surface area, showed significant decreases in left ventricular volume and function in patients compared to controls, although these values remained within normal ranges. Subclinical (but significant) impairment of right ventricular function was observed in patients with either limited or diffuse SSc.

The authors conclude that cardiac MRI shows promise as a rapid, noninvasive method for detecting latent right ventricular impairment, which might otherwise remain undetected in patients with SSc.

Original article Bezante GP et al. (2007) Cardiac magnetic resonance imaging detects subclinical right ventricular impairment in systemic sclerosis. J Rheumatol 34: 2431-2437

\section{Adalimumab therapy decreases fatigue in patients with RA}

Fatigue is a high-priority symptom in patients with rheumatoid arthritis (RA), owing to its markedly detrimental effects on quality of life. Nonetheless, fatigue is rarely assessed by rheumatologists in the clinic or in RA studies. Yount and colleagues assessed the effects of adalimumab therapy on fatigue in patients with RA.

Data were obtained from three randomized, double-blind, placebo-controlled trials of adalimumab therapy. Fatigue was measured by the 13-item scale of the FACIT (functional assessment of chronic illness therapy) questionnaire, which has been validated in RA.

In the ARMADA trial, 271 patients with active RA were randomly allocated to receive methotrexate plus fortnightly adalimumab $20 \mathrm{mg}$, $40 \mathrm{mg}$ or $80 \mathrm{mg}$, or placebo. At 24 weeks, the $40 \mathrm{mg}$ and $80 \mathrm{mg}$ adalimumab groups had significantly improved FACIT fatigue scores compared to the placebo group. In the DE019 trial, in which 496 patients received methotrexate plus either adalimumab $(20 \mathrm{mg}$ weekly or $40 \mathrm{mg}$ fortnightly) or placebo, both treatment groups had significantly improved fatigue scores compared with placebo at all time points (12, 24 and 52 weeks). The STAR trial included 636 patients who received standard therapy (one or more DMARDs, NSAIDs or corticosteroids) plus either fortnightly adalimumab $40 \mathrm{mg}$ or placebo. The treatment group had significantly better FACIT fatigue scores than the placebo group at 12 and 24 weeks.
The authors conclude that $<1$ year of adalimumab therapy significantly improves fatigue in patients with $R A$.

Original article Yount S et al. (2007) Adalimumab plus methotrexate or standard therapy is more effective than methotrexate or standard therapies alone in the treatment of fatigue in patients with active, inadequately treated rheumatoid arthritis. Clin Exp Rheumatol 25: 838-846

\section{Surgery is superior to nonsurgical treatment for spinal stenosis}

Lumbar surgery is widely used to treat spinal stenosis; however, there is little reliable evidence that surgical therapy for this condition provides better outcomes than nonsurgical management. In a 2-year, multicenter US study, Weinstein et al. directly compared the efficacy of surgical and nonsurgical treatment for isolated spinal stenosis. Their results suggest that surgery is indeed superior to nonsurgical treatment.

The study population comprised 654 patients. Of the 289 patients who agreed to randomization, 138 were assigned to surgery and 151 to usual care-active physical therapy, counseling, home exercise instruction and NSAIDs. The remaining patients (the observational cohort) declined randomization; 219 initially chose surgery and 146 chose usual care. However, a considerable number of patients allocated to surgery chose to delay their operation, and many patients allocated to nonsurgical treatment crossed over to undergo surgery. In the randomization cohort, $67 \%$ of patients allocated to surgery had undergone surgery after 2 years, and $43 \%$ of those allocated to nonsurgical treatment had also undergone surgery. In the observational cohort, $96 \%$ of patients who opted for surgery had undergone the operation at 2 years, as had $22 \%$ of those who originally opted for usual care.

Intention-to-treat analysis showed a significant benefit for surgery at 2 years on the Short Form 36 bodily pain score. A combined (as-treated) analysis significantly favored surgery in scores for bodily pain, physical function, and the Oswestry Disability Index at 3 months; these changes remained significant at 2 years.

Original article Weinstein JN et al. (2008) Surgical versus nonsurgical therapy for lumbar spinal stenosis. N Engl J Med 358: 794-810 